Physical Review A, 1988, Volume 37, Issue 11, Pages 4118-4124 (C) 2010 by: The American Physical Society.

ISSN: 1050-2947

http://link.aps.org/

http://pra.aps.org/ 


\title{
Formation of ground and excited states of antihydrogen
}

\author{
Sultana N. Nahar and J. M. Wadehra \\ Department of Physics and Astronomy, Wayne State University, Detroit, Michigan 48202
}

(Received 20 November 1987)

\begin{abstract}
Differential and integrated cross sections for the formation of antihydrogen by the impact of intermediate-energy $(20-500 \mathrm{keV})$ antiprotons on positronium are calculated using the first Born approximation. The calculations are carried out for the formation of antihydrogen in ground and various excited electronic states $(n=1-3)$ when positronium, the target atom, is in the ground state, and for the formation of antihydrogen in the ground state when the positronium is in various excited electronic states $(n=1-2)$. The $1 / n^{3}$ behavior for the capture cross sections is used to calculate the total (that is, all states added together) integrated cross sections. The cross sections for the formation of antihydrogen presented here are obtained from those for the formation of positronium by the impact of positrons on hydrogen atoms by using charge invariance and the principle of detailed balance.
\end{abstract}

\section{INTRODUCTION}

Because of the recent availability of an antiproton beam at the Low-Energy Antiproton Ring (LEAR) facility at Organisation Européenne pour la Recherche Nucleaire (CERN), experiments for the formation of one of the simplest atoms of antimatter, namely, antihydrogen $(\overline{\mathrm{H}})$, are being proposed and planned. ${ }^{1-4}$ One reason for the strong interest in the formation of antihydrogen is its relative stability compared to other exotic atoms such as muonium, protonium, positronium, etc., which makes it more suitable for carrying out experiments with antihydrogen for various diagnostic purposes. In the present work the cross sections are calculated for the formation of $\overline{\mathrm{H}}$ by positron $\left(e^{+}\right)$capture during the collisions of intermediate-energy $(20-500 \mathrm{keV})$ antiprotons $(\bar{p})$ with positronium (Ps) using the first Born approximation (FBA). Schematically the processes are

$$
\bar{p}+\mathbf{P s}(1 s) \rightarrow \overline{\mathbf{H}}(n l m)+e^{--},
$$

where $n l m=1 s_{0}, 2 s_{0}, 2 p_{0}, 2 p_{ \pm 1}, 3 s_{0}, 3 p_{0}, 3 p_{ \pm 1}, 3 d_{0}$, $3 d_{ \pm 1}$, and $3 d_{ \pm 2}$, and

$$
\bar{p}+\mathrm{Ps}(n l m) \rightarrow \overline{\mathrm{H}}(1 s)+e^{-},
$$

where $n l m=1 s_{0}, 2 s_{0}, 2 p_{0}$, and $2 p_{ \pm 1}$. The $1 / n^{3}$ behavior for the capture cross sections in the Born approximation is used to estimate the differential and integrated cross section for the formation of higher excited states of antihydrogen starting from the ground state of Ps as well as for the formation of ground state of $\overline{\mathrm{H}}$ starting from the higher excited states of Ps.

\section{THEORY}

Following earlier works, ${ }^{2,3}$ the cross sections $\left(\sigma_{\overline{\mathrm{H}}}\right)$ for the formation of $\overline{\mathbf{H}}$ by the impact of $\bar{p}$ on Ps of Eqs. (1) and (2) are, by charge invariance, the same as those for the formation of hydrogen $(\mathrm{H})$ by the impact of protons $(p)$ on positronium, that is,

$$
\sigma_{\overline{\mathbf{H}}}=\sigma\left(\bar{p}+\mathbf{P s} \rightarrow \overline{\mathbf{H}}+e^{-}\right)=\sigma\left(p+\mathbf{P s} \rightarrow \mathbf{H}+e^{+}\right) .
$$

Furthermore, the cross sections for the formation of $\mathbf{H}$ by the impact of protons on Ps are related, by the principle of detailed balance, to the cross sections for the formation of Ps by the impact of positrons on hydrogen. Hence one can write

$$
\sigma_{\overline{\mathrm{H}}}=\sigma\left(p+\mathrm{Ps} \rightarrow \mathrm{H}+e^{+}\right)=\frac{k_{i}^{2}}{k_{f}^{2}} \sigma_{\mathrm{Ps}} .
$$

$\sigma_{\mathrm{P}_{\mathrm{s}}}$ is the cross section for the process

$$
e^{+}+\mathrm{H}(n l m) \rightarrow \mathrm{Ps}(1 s)+p,
$$

or for the process

$$
e^{+}+\mathrm{H}(1 s) \rightarrow \operatorname{Ps}(n l m)+p .
$$

$\hbar \mathbf{k}_{i}$ and $\hbar \mathbf{k}_{f}$ are the relative momenta of the positron (in the initial channel) and the positronium (in the final channel) of Eqs. (5). In the present work, the values of $\sigma_{\mathrm{Ps}}$ for the processes of Eqs. (5) for various sets of $n l m$ are calculated first, and then Eq. (4) is used to obtain the values of the cross sections $\left(\sigma_{\overline{\mathrm{H}}}\right)$ for the formation of antihydrogen which are now labeled $\sigma_{n i m}$.

The coordinate system used for calculating $\sigma_{\mathrm{Ps}}$ of Eqs. (5) is shown in Fig. 1. It is clear from Fig. 1 that $\mathbf{r}_{i}\left(\mathbf{r}_{f}\right)$ and $\mathbf{R}_{i}\left(\mathbf{R}_{f}\right)$ are the internal coordinate and the coordinate of the center of mass of the bound system in the ini-

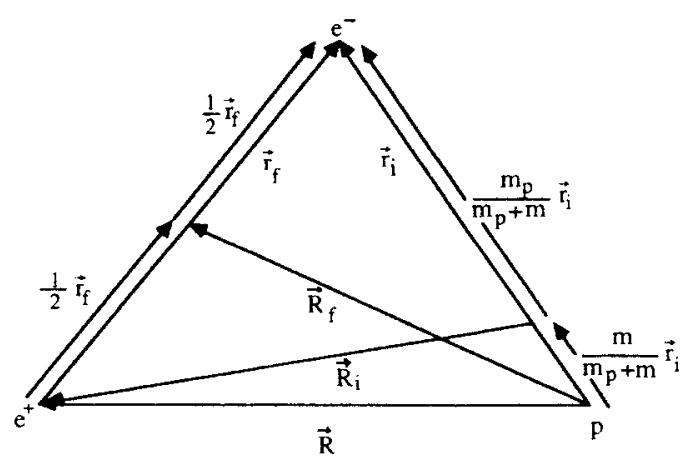

FIG. 1. The coordinate system for the processes of Eqs. (5). 
tial (final) channel, respectively. $\mathbf{R}$ locates the incoming positron with respect to the proton. Atomic units are used throughout this paper unless specified otherwise. To obtain the impact energy of the positron for the processes of Eqs. (5) corresponding to an impact energy of the antiproton for the processes of Eqs. (1) and (2), conservation of momentum is used. Let $E=k_{\bar{p}}^{2} / 2 m_{p}$ be the laboratory impact energy of the antiproton of mass $m_{p}$ with respect to the positronium at rest. Then the wave number $k_{f}$ of the positronium with respect to the stationary antiproton is

$$
k_{f}=m_{\mathrm{Ps}} k_{\bar{p}} / m_{p}
$$

where $m_{\mathrm{Ps}}$ is the mass of the positronium. Hence, in the frame of a stationary antiproton, the energy of the positronium is $k_{f}^{2} /\left(2 m_{\mathrm{Ps}}\right)$. The wave number $k_{i}$ of the positron, of Eqs. (5), is obtained from the conservation of energy, namely,

$$
k_{i}^{2} /\left(2 v_{i}\right)=k_{f}^{2} /\left(2 v_{f}\right)-\varepsilon_{f}+\varepsilon_{i},
$$

where $\varepsilon_{i}$ and $\varepsilon_{f}$ are the (positive) binding energies of $H$ and Ps, respectively. If $m$ is the mass of an electron (or a positron), then $v_{i}=m\left(m+m_{p}\right) /\left(2 m+m_{p}\right)$ is the reduced mass of the system $\left(e^{+}+\mathrm{H}\right)$ in the initial channel and $v_{f}=2 m_{p} m /\left(2 m+m_{p}\right)$ is the reduced mass of the system $(\mathrm{Ps}+p)$ in the final channel of Eqs. (5). In the first Born approximation, the differential cross section for the processes (5) is related to the $T$-matrix element as

$$
\frac{d \sigma}{d \Omega}=\frac{v_{i} v_{f}}{(2 \pi)^{2}} \frac{k_{f}}{k_{i}}|T|^{2},
$$

where

$$
T=\left\{\begin{array}{l}
\left\langle\psi_{f}\left|V_{i}\right| \psi_{i}\right\rangle \text { in the prior form }, \\
\left\langle\psi_{f}\left|V_{f}\right| \psi_{i}\right\rangle \text { in the post form } .
\end{array}\right.
$$

$\psi_{i}$ and $\psi_{f}$ are the wave functions for the system $\left(e^{+}, \mathbf{H}\right)$ in the initial channel and the system (Ps,p) in the final channel, respectively; that is,

$$
\psi_{i}=\exp \left(i \mathbf{k}_{i} \cdot \mathbf{R}_{i}\right) \phi_{i}\left(\mathbf{r}_{i}\right)
$$

and

$$
\psi_{f}=\exp \left(i \mathbf{k}_{f} \cdot \mathbf{R}_{f}\right) \phi_{f}\left(\mathbf{r}_{f}\right)
$$

$$
\begin{aligned}
\phi_{i}\left(3 d_{0}, \mathbf{r}\right) & =R_{32}(r)\left[\frac{5}{16 \pi}\right]^{1 / 2}\left(3 \cos ^{2} \theta-1\right) \\
& =\rho\left(3 d_{0}, r\right)\left[\frac{z^{2}}{r^{2}}-\frac{1}{3}\right] \\
& =\left(-\frac{d^{2}}{d A_{z}^{2}}\left[\exp (i \mathbf{A} \cdot \mathbf{r}) \rho\left(3 d_{0}, r\right) / r^{2}\right]-\exp (i \mathbf{A} \cdot \mathbf{r}) \rho\left(3 d_{0}, r\right) / 3\right]_{\mathbf{A}=0} \\
& =\left(-\frac{d^{2}}{d A_{z}^{2}}\left[\chi_{i}\left(3 d_{0}, \mathbf{r}\right) / r^{2}\right]-\chi_{i}\left(3 d_{0}, \mathbf{r}\right) / 3\right]_{\mathbf{A}=0},
\end{aligned}
$$

where $\phi_{i}\left(\mathbf{r}_{i}\right)$ and $\phi_{f}\left(\mathbf{r}_{f}\right)$ are the bound-state wave functions of $\mathbf{H}$ and Ps, respectively. The initial and the final interaction potentials $V_{i}$ and $V_{f}$ are

$$
\begin{aligned}
& V_{i}=V(R)-V\left(r_{f}\right), \\
& V_{f}=V(R)-V\left(r_{i}\right),
\end{aligned}
$$

where $V(x)=1 / x$. The integrated cross section is given by

$$
\sigma=2 \pi \int_{0}^{\pi}\left[\frac{d \sigma}{d \Omega}\right) \sin \theta d \theta
$$

Substituting $V_{i}$ and $V_{f}$ from Eqs. (11) in the $T$-matrix elements, we get

$$
T=\left\{\begin{aligned}
\left\langle\psi_{f}|V(R)| \psi_{i}\right\rangle- & \left\langle\psi_{f}\left|V\left(r_{f}\right)\right| \psi_{i}\right\rangle \\
& =N_{1}+N_{2} \quad \text { (prior form) } \\
\left\langle\psi_{f}|V(R)| \psi_{i}\right\rangle- & \left\langle\psi_{f}\left|V\left(r_{i}\right)\right| \psi_{i}\right\rangle \\
= & N_{1}+N_{3} \quad \text { (post form) }
\end{aligned}\right.
$$

These $T$-matrix terms, $N_{1}, N_{2}$, and $N_{3}$, can be evaluated ${ }^{5}$ easily for the cases when only the spherically symmetric states are involved. Such cases, for example, are the formation of Ps(1s) from different $s$ states of $\mathrm{H}$ of Eq. (5a) or the formation of different $s$ states of Ps from $\mathrm{H}(1 s)$ of $\mathrm{Eq}$. (5b). Complexity arises when the initial or the final bound-state wave functions in Eqs. (5) are not spherically symmetric as in the case of formation of Ps in the $p$ or $d$ state or when the target $H$ is in an excited $p$ state. The complexity due to the nonspherical wave functions can be reduced by expressing the angular dependence of the wave functions in terms of derivatives of an exponential factor $\exp (i \mathbf{A} \cdot \mathbf{r})$, where the value of the parameter $\mathbf{A}$ is eventually set to zero. In particular, for the processes (5a) we introduce a function $\chi_{i}(r)$ as follows:

$$
\chi_{i}(n l m, \mathbf{r})=\rho(n l m, r) \exp (i \mathbf{A} \cdot \mathbf{r}),
$$

where $\rho(n l m, r)$ represents $R_{n l}$, the radial part of the wave function of hydrogen, multiplied by the constants of $Y_{l m}$, the angular part of the wave function. Then the complete bound-state wave functions $\phi_{i}(r)$, of Eq. (10), can be obtained by merely taking the appropriate derivatives of $\chi_{i}(\mathbf{r})$ with respect to either $A_{x}$ or $A_{y}$ or $A_{z}$ and then setting $\mathbf{A}=0$. As an example, for the $3 d_{0}$ state of hydrogen, the wave function is 
where, using the explicit form of $R_{32}$,

$$
\rho\left(3 d_{0}, r\right)=\frac{1}{27 \sqrt{6 \pi}} r^{2} \exp (-r / 3) .
$$

This kind of trick has been used earlier by Sil et al. ${ }^{6}$ in calculations of charge-transfer cross sections.

Let $\mathbf{Q}_{i}$ and $\mathbf{Q}_{f}$ be the momentum-transfer vectors in the initial and final channel, respectively, i.e.,

$$
\mathbf{Q}_{i}=\left[m_{p} /\left(m+m_{p}\right)\right] \mathbf{k}_{i}-\mathbf{k}_{f}, \quad \mathbf{Q}_{f}=\mathbf{k}_{f} / 2-\mathbf{k}_{i}
$$

and let $\mu$ be the reduced mass of the final atom positronium of the processes (5). Then the parts of the $T$-matrix elements for the processes (5a), upon replacing $\phi_{i}(\mathbf{r})$ by $\chi_{i}(n l m, \mathbf{r})$ of Eq. (14), can be written as

$$
\begin{aligned}
& N_{1}=(2 \pi)^{3 / 2} \int \tilde{\phi}_{f}^{*}\left(\mathbf{Q}_{f}-\mathbf{t}\right) \widetilde{v}(\mathbf{t}) \tilde{\rho}\left(-\mathbf{t}-\mathbf{Q}_{i}-\mathbf{A}\right) d^{3} t, \\
& N_{2}=-(2 \pi)^{3}\left[\left(\frac{Q_{f}^{2}}{2 \mu}+\varepsilon_{f}\right] \tilde{\phi}_{f}^{*}\left(\mathbf{Q}_{f}\right) \tilde{\rho}\left(-\mathbf{Q}_{i}-\mathbf{A}\right)\right], \\
& N_{3}=-(2 \pi)^{3 / 2} \tilde{\phi}_{f}^{*}\left(\mathbf{Q}_{f}\right) \int \tilde{v}(\mathbf{t}) \tilde{\rho}\left(-\mathbf{t}-\mathbf{Q}_{i}-\mathbf{A}\right) d^{3} t,
\end{aligned}
$$

where the overtilde represents the Fourier transform of the respective function. For example, the Fourier transform $\widetilde{f}(\mathbf{t})$ of a function $f(\mathbf{r})$ is defined as

$$
\tilde{f}(\mathbf{t})=(2 \pi)^{-3 / 2} \int d^{3} r \exp (-i \mathbf{t} \cdot \mathbf{r}) f(\mathbf{r}) .
$$

Substituting the explicit forms for the potential $V$, and the wave functions $\phi_{i}$ and $\phi_{f}$, the term $N_{1}$ incurs an integral of the form

$$
I_{1}=\int d^{3} t \frac{1}{(\mathbf{t}-\mathbf{P})^{2}+p^{2}} \frac{1}{(\mathbf{t}-\mathbf{B})^{2}+b^{2}} \frac{1}{t^{2}+z^{2}},
$$

which, upon using the Feynman identity, can be reduced to a one-dimensional integral as ${ }^{5}$

$$
I_{1}=\pi^{2} \int_{0}^{1} \frac{d x}{E\left[F^{2}+(E+z)^{2}\right]},
$$

where $\quad E^{2}=x(1-x)(\mathbf{P}-\mathbf{B})^{2}+x p^{2}+(1-x) b^{2} \quad$ and $\mathbf{F}=(\mathbf{P}-\mathbf{B}) x+\mathbf{B} . N_{2}$ can be written in a pure analytic fashion. The term $\mathrm{N}_{3}$ involves integral of the form

$$
I_{3}=\int d^{3} t \frac{1}{\left[(\mathbf{t}-\mathbf{P})^{2}+p^{2}\right]^{2}} \frac{1}{t^{2}+z^{2}}
$$

which can be done analytically to obtain

$$
I_{3}=\pi^{2} \frac{1}{p\left[P^{2}+(p+z)^{2}\right]} .
$$

Integral $I_{3}$ of Eq. (21) easily follows from integral $I_{1}$ of Eq. (19) by setting $\mathbf{P}=\mathbf{B}$ and $p=b . \mathbf{P}, \mathbf{B}, p, b$, and $z$ of Eqs. (19) and (21) are, of course, related to $\mathbf{Q}_{i}, \mathbf{Q}_{f}$ and the parameter $\mathbf{A}$ of Eqs. (18a) and (18c). In the case of $p$ and $d$-state wave functions, $N_{1}, N_{2}$, and $N_{3}$ will involve derivatives with respect to the Cartesian components of A. Note that since $\phi_{n l m}$ is a complex conjugate of $\phi_{n l-m}$, and $|T|^{2}$ is used to calculate the cross section, the cross sections with both the wave functions $\left(\phi_{n l \pm m}\right)$ are the same. This technique of using the derivatives of the factor $\exp (i \mathbf{A} \cdot \mathbf{r})$ for representing the angular dependence of the wave functions can also be used for the for- mation of various excited states of Ps from $\mathrm{H}(1 s)$ of Eq. (5b). In this case the factor $\exp (i \mathbf{A} \cdot \mathbf{r})$ is introduced in the final bound-state wave function $\phi_{f}(\mathbf{r})$ rather than in the initial bound-state wave function $\phi_{i}(\mathbf{r})$. Thus after calculating the cross sections for the processes of Eqs. (5), the corresponding cross sections for the formation of antihydrogen are obtained using Eq. (4).

It was predicted by Oppenheimer ${ }^{7}$ that the cross section for capture into any $n s$ excited hydrogenic state at high incident energies falls as $1 / n^{3}$. Later, Omidvar ${ }^{8}$ showed that the cross section for the capture into $s, p$, and $d$ states as well as the sum of the cross sections over Im states falls as $1 / n^{3}$ in the first Born approximation. There is also an experimental evidence of the cross section for electron capture into excited states falling as $1 / n^{3}$ in collisions of high-energy fluorine ions with argon. ${ }^{9}$ Hence using the $1 / n^{3}$ dependence of the capture cross sections of Eq. (1) for the formation of $\mathrm{H}$ in excited states $(n \geq 4)$, the total cross section could be written as

$$
\begin{gathered}
\sigma_{T}=\sigma_{1}+\sigma_{2}+\sigma_{3}\left[1+\left(\frac{n}{n+1}\right)^{3}+\left(\frac{n}{n+2}\right)^{3}\right. \\
\left.+\left(\frac{n}{n+3}\right]^{3}+\cdots\right),
\end{gathered}
$$

where

$$
\begin{aligned}
& \sigma_{1}=\sigma_{1 s_{0}}, \\
& \sigma_{2}=\sigma_{2 s_{0}}+\sigma_{2 p_{0}}+2 \sigma_{2 p_{1}}, \\
& \sigma_{3}=\sigma_{3 s_{0}}+\sigma_{3 p_{0}}+2 \sigma_{3 p_{1}}+\sigma_{3 d_{0}}+2 \sigma_{3 d_{1}}+2 \sigma_{3 d_{2}},
\end{aligned}
$$

and $n=3$. The value of the series within the large square brackets of Eq. (23) is 2.0805 for $n=3$. In the case of $\mathbf{H}(1 s)$ formation from various states of Ps, of Eq. (2), the $1 / n^{3}$ rule is used for positronium states $n \geq 3$. Hence using $n=2$ the value of the series within the large square brackets of Eq. (23) is 1.616 .

\section{RESULTS AND DISCUSSIONS}

The present work on charge-transfer processes has been carried out using both the prior and the post forms of the interaction. The difference in the values of the cross sections using these two forms of the interaction (that is, the post-prior discrepancy) is quite small (a few parts in a thousand). Hence only the prior form of the results is presented. The $n^{-3}$ behavior for the capture cross sections is used for antihydrogen formation in states $n \geq 4$ from $\mathrm{Ps}(1 s)$ [see Eq. (1)] and for $\overline{\mathbf{H}}(1 s)$ formation from Ps in states $n \geq 3$ [see Eq. (2)]. The differential cross sections (DCS's) for the formation of antihydrogen in all states (ground and all possible excited states added together) by the impact of antiprotons on positronium in ground state are shown in Fig. 2 and those for the formation of antihydrogen in the ground state from all possible states of positronium are shown in Fig. 3. From Figs. 2 and 3 we see that the nature of the DCS curves in these two cases are very similar. DCS curves in Fig. 2 show 
that following the forward peak there is a very shallow minimum at lower energies that deepens with increasing antiproton impact energies while its position stays at roughly the same angle $\left(\sim 24^{\circ}\right)$. At larger scattering angles, the values of DCS's fall smoothly at all energies. Similar is the case with the curves of Fig. 3, except that the minima in the DCS values at lower impact energies are more pronounced than those of Fig. 2. The minima in the DCS curves arise from the opposite nature (attractive versus repulsive) of antiproton-positron and antiproton-electron interactions; essentially the $N_{1}$ and the $N_{2}$ parts of the $T$-matrix element [Eq. (13)] cancel one another. The integrated cross sections for the formation of antihydrogen in individual states $1 s_{0}, 2 s_{0}, 2 p_{0}, 2 p_{1}$, $3 s_{0}, 3 p_{0}, 3 p_{1}, 3 d_{0}, 3 d_{1}, 3 d_{2}$, as well as in all possible states, using the $n^{-3}$ behavior, from Ps(1s), are numerically presented in Table $I$ and are shown in Fig. 4. The integrated cross sections for the formation of $\overline{\mathrm{H}}(1 s)$ from
Ps in individual states $1 s_{0}, 2 s_{0}, 2 p_{0}, 2 p_{1}$, as well as in all possible states, using the $n^{-3}$ behavior, are numerically presented in Table II and are shown in Fig. 5. All the curves for integrated cross sections in Figs. 4 and 5 decay very smoothly with increasing $\vec{p}$ impact energies. From Table I and Fig. 4 we see that formation of the $p$ and $d$ states of antihydrogen dominates over the formation of any other state at lower antiproton impact energy $(<50$ $\mathrm{keV})$. However, at higher impact energies the formation of $\overline{\mathbf{H}}(1 s)$ dominates over the formation of all other states. In case of formation of $\overline{\mathrm{H}}(1 s)$ from various states of Ps, as seen in Table II and Fig. 5, the formation from $\mathrm{Ps}(1 s)$ dominates significantly over formation from any other state of positronium almost at all energies considered.

The use of $1 / n^{3}$ behavior of the capture cross sections for the entire range of antiproton impact energies to calculate the cross sections for states $n \geq 4$ for processes of Eq. (1) and for states $n \geq 3$ for processes of Eq. (2) is

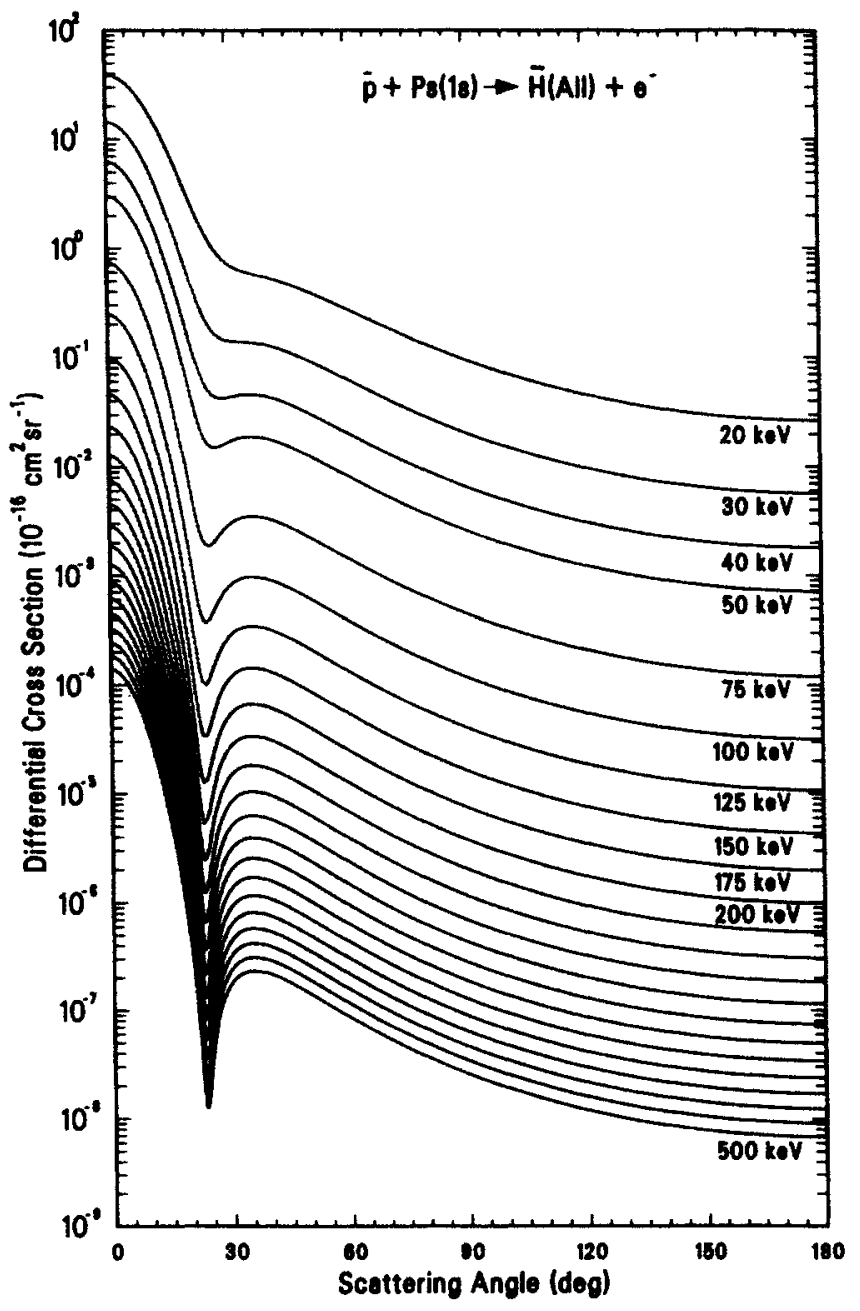

FIG. 2. The differential cross sections for the formation of antihydrogen in all possible states by the impact of antiprotons on Ps(1s), Eq. (1). The DCS curves between 200 and $500 \mathrm{keV}$ correspond to sequential increase of impact energy at an interval of $25 \mathrm{keV}$.

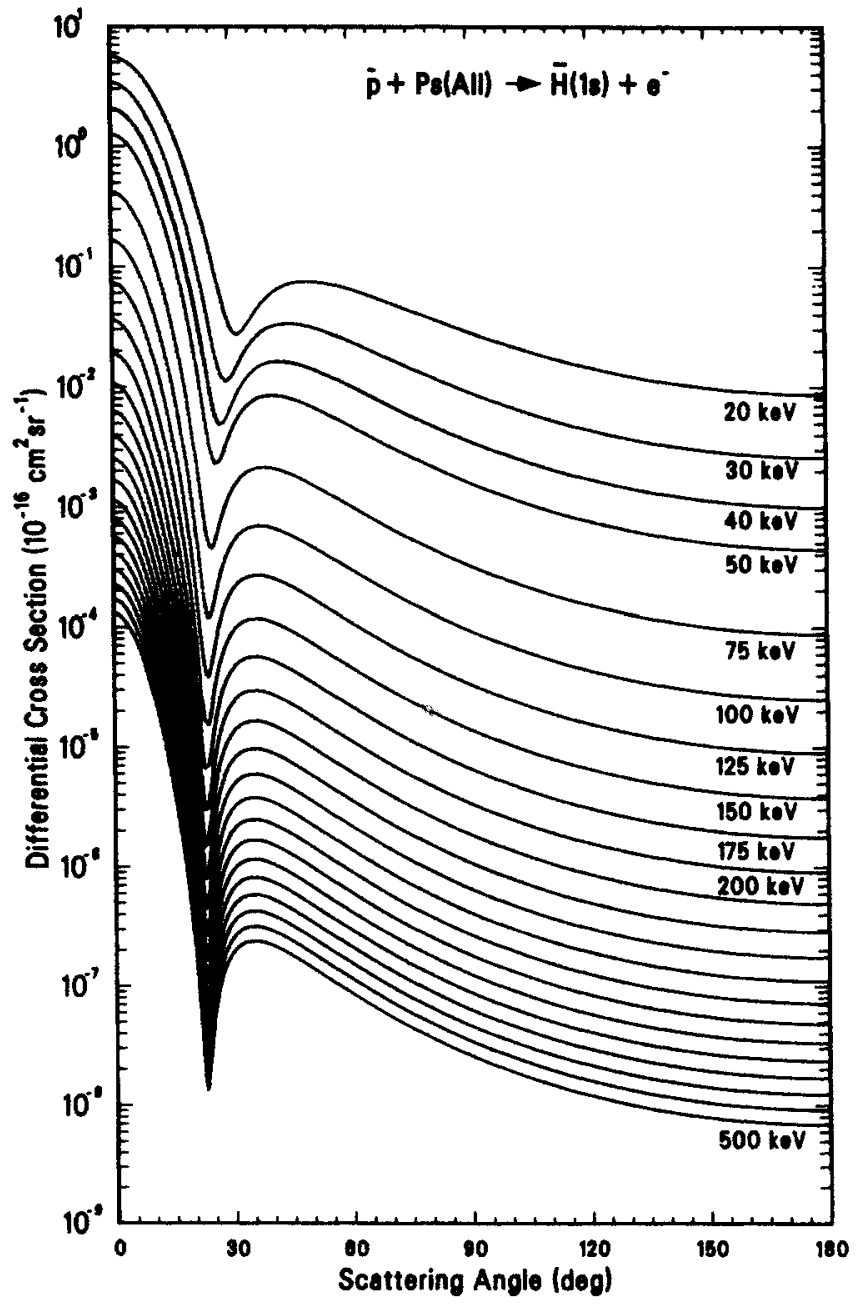

FIG. 3. The differential cross sections for the formation of antihydrogen in the ground state by the impact of antiprotons on all possible states of positronium, Eq. (2). The DCS curves between 200 and $500 \mathrm{keV}$ correspond to sequential increase of impact energy at an interval of $25 \mathrm{keV}$. 
found to be quite reasonable. For example, the value of the ratio $(n+1)^{3} \sigma_{n+1} / n^{3} \sigma_{n}$, which ideally should be 1 if the $1 / n^{3}$ behavior is valid, is 1.54 at $20 \mathrm{keV}$ and 1.07 at $500 \mathrm{keV}$ for the process of Eq. (1) when $n=2$. The same ratio for the process of Eq. (2) is 1.26 at $20 \mathrm{keV}$ and 1.02 at $500 \mathrm{keV}$ for $n=1$. These numbers suggest that the use of $1 / n^{3}$ rule holds relatively more accurately at higher impact energies than at lower impact energies.

In the earlier works, Neuman et al. ${ }^{1}$ have carried out a laser-enhanced electron-ion-capture calculation to evaluate the rate of total recombination for antihydrogen formation. Humberston et al. ${ }^{2}$ presented the values of cross sections for the formation of $\overline{\mathbf{H}}(1 s)$ from $\operatorname{Ps}(1 s)$ at lower impact energies using the $s-, p$-, and $d$-wave positronium- formation phase shifts obtained by elaborate variational calculations. Later, Darewych ${ }^{3}$ calculated the cross sections for the formation of $\overline{\mathrm{H}}$ in states $1 s_{0}, 2 s_{0}, 2 p$ (all $\mathrm{m}$ ), and $3 s_{0}$ from Ps( $\left.1 s\right)$ at low antiproton impact energies using the first Born approximation (FBA). Since the FBA is not a good approximation for scattering calculations at low projectile energies, the present work is carried out in an intermediate range of impact energies. However, for computational checking purposes, cross sections at a few low impact energies were calculated and it was observed that both Ref. 3 and the present work agree very well for the formation of $\overline{\mathbf{H}}$ in $s$ states, while the values of the cross sections for $2 p$ - (all $m$ ) state formation in the present work are much larger than those of Ref. 3. As a

TABLE I. The values of the integrated cross sections $\left(\sigma_{\overline{\mathrm{H}}}\right)$ for the formation of $\overline{\mathrm{H}}$ in ground and various excited states as well as in all possible states by the impact of $\bar{p}$ on $\mathrm{Ps}(1 s)$ in the incident-energy range of $E=20-500 \mathrm{keV}$. The notation a[b] for the values of the cross sections means $a \times 10^{b}$.

\begin{tabular}{|c|c|c|c|c|c|c|c|c|}
\hline \multirow[b]{2}{*}{ States } & \multirow[b]{2}{*}{$E=20$} & \multicolumn{6}{|c|}{$\sigma_{\overline{\mathrm{H}}}\left(10^{-16} \mathrm{~cm}^{2}\right)$ at various $\bar{p}$ energies, $E(\mathrm{keV})$} & \multirow[b]{2}{*}{150} \\
\hline & & 30 & 40 & 50 & 75 & 100 & 125 & \\
\hline $1 s_{0}$ & $0.1048[1]$ & 0.4640 & 0.2302 & 0.1242 & $0.3425[-1]$ & $0.1208[-1]$ & $0.5025[-2]$ & $0.2352[-2]$ \\
\hline $2 s_{0}$ & 0.2780 & 0.1109 & $0.5372[-1]$ & $0.2837[-1]$ & $0.7344[-2]$ & $0.2438[-2]$ & $0.9624[-3]$ & $0.4315[-3]$ \\
\hline $2 p_{0}$ & 0.9554 & 0.2794 & $0.9847[-1]$ & $0.3994[-1]$ & $0.6384[-2]$ & $0.1516[-2]$ & $0.4643[-3]$ & $0.1697[-3]$ \\
\hline $2 p_{1}$ & 0.4057 & 0.1001 & $0.3150[-1]$ & $0.1176[-1]$ & $0.1643[-2]$ & $0.3593[-3]$ & $0.1040[-3]$ & $0.3646[-4]$ \\
\hline $3 s_{0}$ & 0.1149 & $0.4015[-1]$ & $0.1862[-1]$ & $0.9648[-2]$ & $0.2439[-2]$ & $0.7973[-3]$ & $0.3112[-3]$ & $0.1383[-3]$ \\
\hline $3 p_{0}$ & 0.3173 & 0.1009 & $0.3674[-1]$ & $0.1511[-1]$ & $0.2429[-2]$ & $0.5741[-3]$ & $0.1747[-3]$ & $0.6345[-4]$ \\
\hline $3 p_{1}$ & 0.1264 & $0.3439[-1]$ & $0.1124[-1]$ & $0.4270[-2]$ & $0.6042[-3]$ & $0.1321[-3]$ & $0.3813[-4]$ & $0.1333[-4]$ \\
\hline $3 d_{0}$ & $0.9331[-1]$ & $0.2305[-1]$ & $0.6651[-2]$ & $0.2241[-2]$ & $0.2451[-3]$ & $0.4359[-4]$ & $0.1061[-4]$ & $0.3211[-5]$ \\
\hline $3 d_{1}$ & $0.5982[-1]$ & $0.1323[-1]$ & $0.3564[-2]$ & $0.1144[-2]$ & $0.1157[-3]$ & $0.1961[-4]$ & $0.4613[-5]$ & $0.1360[-5]$ \\
\hline $3 d_{2}$ & $0.1886[-1]$ & $0.3340[-2]$ & $0.7996[-3]$ & $0.2379[-3]$ & $0.2149[-4]$ & $0.3417[-5]$ & $0.7709[-6]$ & $0.2206[-6]$ \\
\hline \multirow[t]{2}{*}{ All } & $0.5039[1]$ & $0.1608[1]$ & 0.6393 & 0.2957 & $0.6499[-1]$ & $0.2035[-1]$ & $0.7873[-2]$ & $0.3514[-2]$ \\
\hline & $E=175$ & 200 & 225 & 250 & 275 & 300 & 325 & 350 \\
\hline $1 s_{0}$ & $0.1204[-2]$ & $0.6611[-3]$ & $0.3843[-3]$ & $0.2340[-3]$ & $0.1483[-3]$ & $0.9710[-4]$ & $0.6546[-4]$ & $0.4525[-4]$ \\
\hline $2 s_{0}$ & $0.2131[-3]$ & $0.1136[-3]$ & $0.6438[-4]$ & $0.3837[-4]$ & $0.2385[-4]$ & $0.1537[-4]$ & $0.1021[-4]$ & $0.6969[-5]$ \\
\hline $2 p_{0}$ & $0.7069[-4]$ & $0.3256[-4]$ & $0.1624[-4]$ & $0.8637[-5]$ & $0.4848[-5]$ & $0.2847[-5]$ & $0.1737[-5]$ & $0.1096[-5]$ \\
\hline $2 p_{1}$ & $0.1471[-4]$ & $0.6604[-5]$ & $0.3225[-5]$ & $0.1686[-5]$ & $0.9324[-6]$ & $0.5405[-6]$ & $0.3262[-6]$ & $0.2038[-6]$ \\
\hline $3 s_{0}$ & $0.6786[-4]$ & $0.3598[-4]$ & $0.2030[-4]$ & $0.1206[-4]$ & $0.7478[-5]$ & $0.4808[-5]$ & $0.3189[-5]$ & $0.2173[-5]$ \\
\hline $3 p_{0}$ & $0.2629[-4]$ & $0.1205[-4]$ & $0.5984[-5]$ & $0.3172[-5]$ & $0.1775[-5]$ & $0.1039[-5]$ & $0.6325[-6]$ & $0.3981[-6]$ \\
\hline $3 p_{1}$ & $0.5360[-5]$ & $0.2399[-5]$ & $0.1169[-5]$ & $0.6096[-6]$ & $0.3364[-6]$ & $0.1946[-6]$ & $0.1173[-6]$ & $0.7315[-7]$ \\
\hline $3 d_{0}$ & $0.1141[-5]$ & $0.4583[-6]$ & $0.2030[-6]$ & $0.9738[-7]$ & $0.4990[-7]$ & $0.2704[-7]$ & $0.1537[-7]$ & $0.9112[-8]$ \\
\hline $3 d_{1}$ & $0.4729[-6]$ & $0.1864[-6]$ & $0.8111[-7]$ & $0.3822[-7]$ & $0.1923[-7]$ & $0.1023[-7]$ & $0.5702[-8]$ & $0.3309[-8]$ \\
\hline $3 d_{2}$ & $0.7495[-7]$ & $0.2901[-7]$ & $0.1244[-7]$ & $0.5791[-8]$ & $0.2884[-8]$ & $0.1520[-8]$ & $0.8408[-9]$ & $0.4846[-9]$ \\
\hline \multirow[t]{2}{*}{ All } & $0.1740[-2]$ & $0.9323[-3]$ & $0.5317[-3]$ & $0.3190[-3]$ & $0.1997[-3]$ & $0.1295[-3]$ & $0.8656[-4]$ & $0.5941[-4]$ \\
\hline & $E=375$ & 400 & 425 & 450 & 475 & 500 & & \\
\hline $1 s_{0}$ & $0.3198[-4]$ & $0.2304[-4]$ & $0.1690[-4]$ & $0.1259[-4]$ & $0.9512[-5]$ & $0.7280[-5]$ & & \\
\hline $2 s_{0}$ & $0.4869[-5]$ & $0.3473[-5]$ & $0.2524[-5]$ & $0.1864[-5]$ & $0.1398[-5]$ & $0.1062[-5]$ & & \\
\hline $2 p_{0}$ & $0.7121[-6]$ & $0.4746[-6]$ & $0.3236[-6]$ & $0.2251[-6]$ & $0.1595[-6]$ & $0.1149[-6]$ & & \\
\hline $2 p_{1}$ & $0.1312[-6]$ & $0.8677[-7]$ & $0.5874[-7]$ & $0.4060[-7]$ & $0.2860[-7]$ & $0.2049[-7]$ & & \\
\hline $3 s_{0}$ & $0.1517[-5]$ & $0.1081[-5]$ & $0.7844[-6]$ & $0.5790[-6]$ & $0.4338[-6]$ & $0.3294[-6]$ & & \\
\hline $3 p_{0}$ & $0.2581[-6]$ & $0.1716[-6]$ & $0.1168[-6]$ & $0.8112[-7]$ & $0.5738[-7]$ & $0.4127[-7]$ & & \\
\hline $3 p_{1}$ & $0.4703[-7]$ & $0.3106[-7]$ & $0.2100[-7]$ & $0.1450[-7]$ & $0.1020[-7]$ & $0.7301[-8]$ & & \\
\hline $3 d_{0}$ & $0.5604[-8]$ & $0.3560[-8]$ & $0.2328[-8]$ & $0.1562[-8]$ & $0.1074[-8]$ & $0.7534[-9]$ & & \\
\hline $3 d_{1}$ & $0.1989[-8]$ & $0.1233[-8]$ & $0.7860[-9]$ & $0.5133[-9]$ & $0.3426[-9]$ & $0.2332[-9]$ & & \\
\hline $3 d_{2}$ & $0.2896[-9]$ & $0.1786[-9]$ & $0.1132[-9]$ & $0.7361[-10]$ & $0.4893[-10]$ & $0.3319[-10]$ & & \\
\hline All & $0.4173[-4]$ & $0.2991[-4]$ & $0.2184[-4]$ & $0.1620[-4]$ & $0.1219[-4]$ & $0.9303[-5]$ & & \\
\hline
\end{tabular}




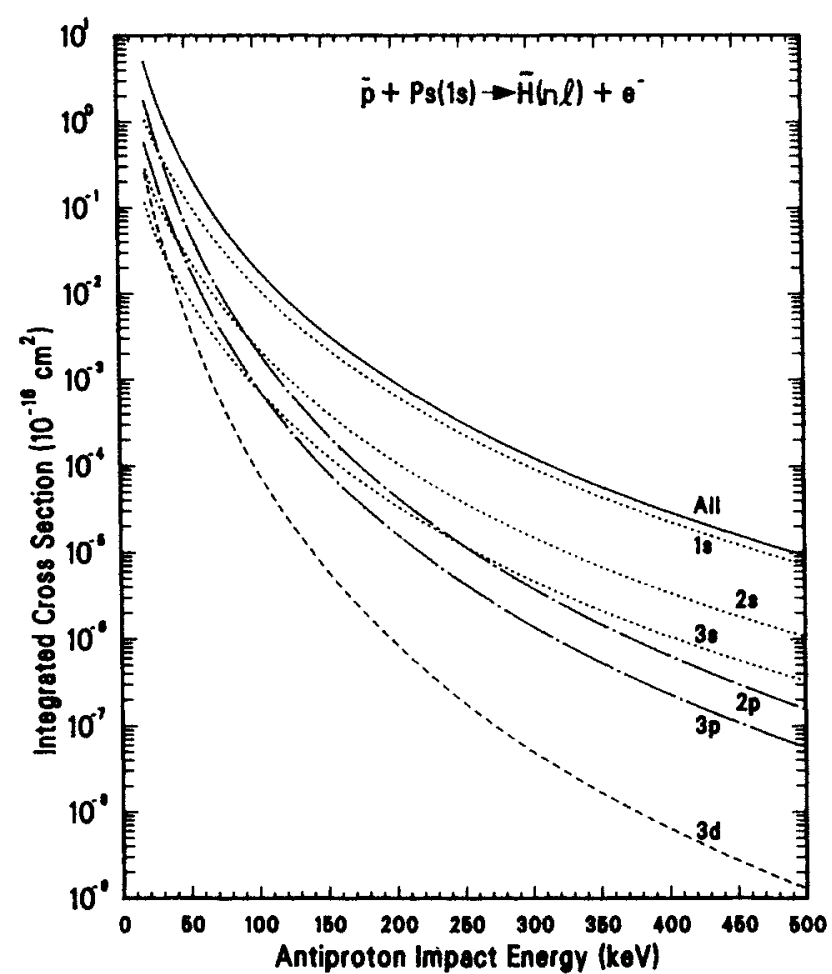

FIG. 4. The integrated cross sections for the formation of antihydrogen in various $s, p$, and $d$ states (summed over $m$ quantum numbers) by the impact of antiprotons on $\operatorname{Ps}(1 s)$, Eq. (1). The solid curve corresponds to the total integrated (i.e., sum of all possible $n l$ states of antihydrogen) cross sections.

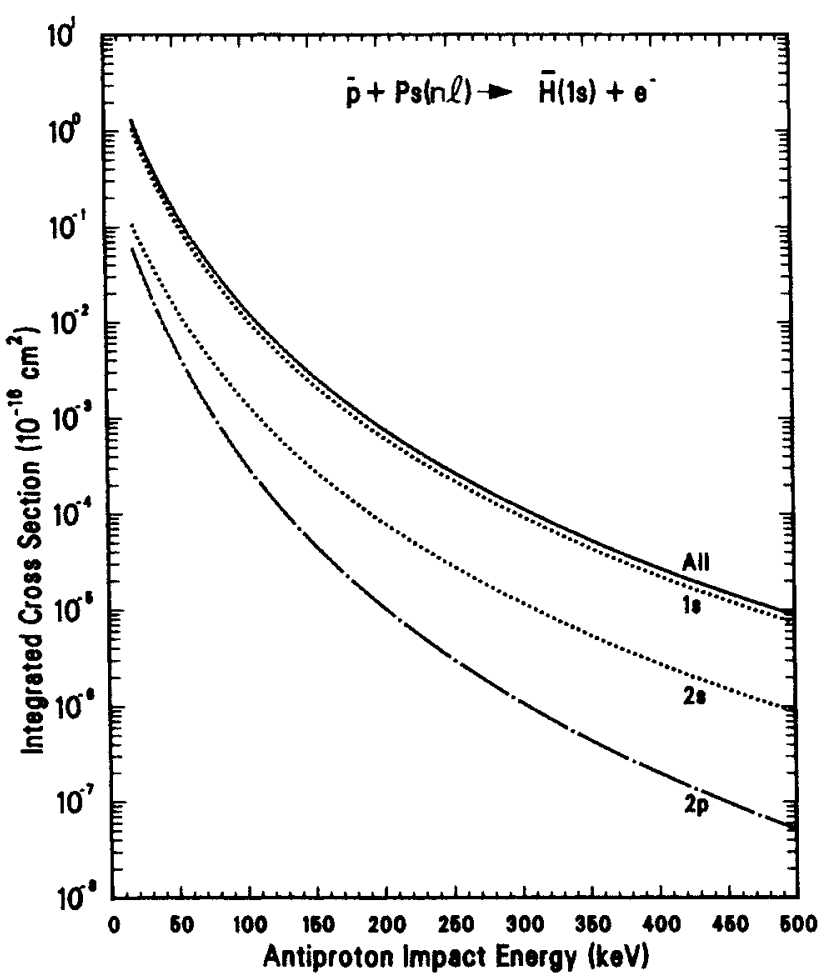

FIG. 5. The integrated cross sections for the formation of antihydrogen in the ground state by the impact of antiprotons on positronium in various $s$ and $p$ states (summed over $m$ quantum numbers), Eq. (2). The solid curve corresponds to the total integrated (i.e., sum of all possible $n l$ states of positronium) cross sections.

TABLE II. The values of the integrated cross sections $\left(\sigma_{\overline{\mathrm{H}}}\right)$ for the formation of $\overline{\mathrm{H}}(1 s)$ by the impact of $\bar{p}$ on ground and various excited states as well as on all possible states of Ps in the incident-energy range of $E=20-500 \mathrm{keV}$. The notation $\mathrm{a}[\mathrm{b}]$ for the values of the cross sections means $a \times 10^{b}$.

\begin{tabular}{|c|c|c|c|c|c|}
\hline \multirow[b]{2}{*}{$E(\mathrm{keV})$} & \multicolumn{5}{|c|}{$\begin{array}{c}\text { Ps state } \\
\sigma_{\overline{\mathrm{H}}}\left(10^{-16} \mathrm{~cm}^{2}\right)\end{array}$} \\
\hline & $1 s_{0}$ & $2 s_{0}$ & $2 p_{0}$ & $2 p_{1}$ & All \\
\hline 20 & $0.1048[1]$ & 0.1054 & $0.3934[-1]$ & $0.1020[-1]$ & $0.1315[1]$ \\
\hline 30 & 0.4640 & $0.5235[-1]$ & $0.1659[-1]$ & $0.4220[-2]$ & 0.5890 \\
\hline 40 & 0.2302 & $0.2757[-1]$ & $0.7753[-2]$ & $0.1877[-2]$ & 0.2933 \\
\hline 50 & 0.1242 & $0.1539[-1]$ & $0.3896[-2]$ & $0.8978[-3]$ & 0.1583 \\
\hline 75 & $0.3425[-1]$ & $0.4408[-2]$ & $0.8889[-3]$ & $0.1854[-3]$ & $0.4341[-1]$ \\
\hline 100 & $0.1208[-1]$ & $0.1572[-2]$ & $0.2622[-3]$ & $0.5093[-4]$ & $0.1521[-1]$ \\
\hline 125 & $0.5025[-2]$ & $0.6545[-3]$ & $0.9291[-4]$ & $0.1711[-4]$ & $0.6288[-2]$ \\
\hline 150 & $0.2352[-2]$ & $0.3054[-3]$ & $0.3773[-4]$ & $0.6665[-5]$ & $0.2928[-2]$ \\
\hline 175 & $0.1204[-2]$ & $0.1555[-3]$ & $0.1702[-4]$ & $0.2907[-5]$ & $0.1492[-2]$ \\
\hline 200 & $0.6611[-3]$ & $0.8490[-4]$ & $0.8342[-5]$ & $0.1387[-5]$ & $0.8163[-3]$ \\
\hline 225 & $0.3843[-3]$ & $0.4903[-4]$ & $0.4375[-5]$ & $0.7106[-6]$ & $0.4729[-3]$ \\
\hline 250 & $0.2340[-3]$ & $0.2967[-4]$ & $0.2427[-5]$ & $0.3864[-6]$ & $0.2872[-3]$ \\
\hline 275 & $0.1483[-3]$ & $0.1867[-4]$ & $0.1411[-5]$ & $0.2208[-6]$ & $0.1814[-3]$ \\
\hline 300 & $0.9710[-4]$ & $0.1216[-4]$ & $0.8544[-6]$ & $0.1316[-6]$ & $0.1186[-3]$ \\
\hline 325 & $0.6546[-4]$ & $0.8146[-5]$ & $0.5355[-6]$ & $0.8132[-7]$ & $0.7975[-4]$ \\
\hline 350 & $0.4525[-4]$ & $0.5599[-5]$ & $0.3459[-6]$ & $0.5187[-7]$ & $0.5503[-4]$ \\
\hline 375 & $0.3198[-4]$ & $0.3936[-5]$ & $0.2295[-6]$ & $0.3401[-7]$ & $0.3882[-4]$ \\
\hline 400 & $0.2304[-4]$ & $0.2823[-5]$ & $0.1558[-6]$ & $0.2285[-7]$ & $0.2793[-4]$ \\
\hline 425 & $0.1690[-4]$ & $0.2060[-5]$ & $0.1081[-6]$ & $0.1569[-7]$ & $0.2046[-4]$ \\
\hline 450 & $0.1259[-4]$ & $0.1528[-5]$ & $0.7639[-7]$ & $0.1099[-7]$ & $0.1522[-4]$ \\
\hline 475 & $0.9512[-5]$ & $0.1150[-5]$ & $0.5492[-7]$ & $0.7829[-8]$ & $0.1149[-4]$ \\
\hline 500 & $0.7280[-5]$ & $0.8768[-6]$ & $0.4010[-7]$ & $0.5668[-8]$ & $0.8781[-5]$ \\
\hline
\end{tabular}


check on the present computer code, the cross sections ${ }^{10}$ for the formation of $\mathrm{H}$, by the process of electron capture during proton-hydrogen collisions, in states $1 s$ and $2 p$ were reproduced.

In conclusion, we have presented a simple first Born calculation of cross sections for the formation of antihydrogen, by the impact of intermediate-energy (20-500
$\mathrm{keV}$ ) antiprotons on positronium, in ground and in various excited states.

\section{ACKNOWLEDGMENT}

We gratefully acknowledge the support of the U.S. Air Force Office of Scientific Research through Grant No. AFOSR -87-0342.
1J. Berger et al., CERN Report No. PSCC 1985, p. 86 (unpublished), (quoted in Ref. 2); R. Neuman, H. Poth, A. Winnacker, and A. Wolf, Z. Phys. A 313, 253 (1983).

$2 J$. W. Humberston, M. Charlton, F. M. Jacobsen, and B. I. Deutch, J. Phys. B 20, L25 (1987).

${ }^{3}$ J. W. Darewych, in Abstracts of the NATO Advanced Reserach Workshop on Atomic Physics with Positrons, University College London, 1987 [J. Phys. B 20, 5917 (1987)].

${ }^{4}$ B. I. Deutch, F. M. Jacobsen, L. H. Andersen, P. Hvelplund, H. Knudsen, M. Holzscheiter, M. Charlton, and G. Laricchia (private communication).
${ }^{5}$ R. Shakeshaft and J. M. Wadehra, Phys. Rev. A 22, 968 (1980); Sultana N. Nahar and J. M. Wadehra, ibid. 35, 4533 (1987).

${ }^{6}$ N. C. Sil, B. C. Saha, H. P. Saha, and P. Mandal, Phys. Rev. A 19, 655 (1979).

${ }^{7}$ J. R. Oppenheimer, Phys. Rev. 31, 349 (1928).

${ }^{8}$ K. Omidvar, Phys. Rev. A 12, 911 (1975).

${ }^{9}$ J. R. Macdonald, P. Richard, C. L. Cocke, M. Brown, and I. A. Sellin, Phys. Rev. Lett. 31, 684 (1973).

${ }^{10}$ D. R. Bates and A. Dalgarno, Proc. Phys. Soc. London, Sect. A 66, 972 (1953). 